\title{
Umbilical cord blood: an expandable resource
}

\author{
Malcolm A.S. Moore
}

Memorial-Sloan-Kettering Cancer Center, New York, New York 10021, USA.

Phone: (212) 639-7090; Fax: (212) 717-3618; E-mail: m-moore@ski.mskcc.org.

Cord blood banks have been established as an alternative source of hematopoietic reconstituting cells for allogeneic transplantation. More than 1,000 cord blood (CB) transplants have been performed from related or unrelated donors, with little or no resulting graftversus-host disease, even in cases with 1 or 2 HLA-antigen mismatches (1). The major limitation to the widespread use of this stem cell source is the low number of cells obtained, which restricts its use in adults. Typically, CB recipients receive 10 -fold fewer $\mathrm{CD}_{3} 4^{+}$cells and progenitors than do recipients of marrow or G-CSF-mobilized peripheral blood stem cells. In individuals 60 to 70 $\mathrm{kg}$. the recovery of neutrophils and, particularly, platelets, is consequently delayed, and grafts fail in $16 \%$ of cases. These data provide a rationale for expanding CB progenitor cells ex vivo. Cord blood expansion studies began 7 years ago, using combinations of ILs (IL-3, IL-1, IL-6) and CSFs (G-CSF, GMCSF) (2). Subsequent studies used c-kit ligand (KL, variously termed steel factor or stem cell factor), Flk2Flt3 ligand (FL)m and thrombopoietin (Tpo) (3-8). These ligands seemed particularly effective in expanding cells with stem cell potential as detected in assays for longterm (5-8 weeks) culture-initiating cells (LTC-ICs) or "cobblestone" area-forming cells (CAFCs) $(3,6,8)$. Dramatic long-term expansion of $\mathrm{CB}$ progenitors and LTC-ICs has been reported by Piacibello et al $(6,7)$, with a combination of FL, Tpo, KL, and IL-6 producing a 100million-fold expansion of progenitors and a 270,000-fold expansion of LTCICs over 7 months.

The utility of existing assays for predicting human long-term engraftment potential of human stem cells is a matter of ongoing debate. Repopulation assays, in which human hematopoietic cells are injected intravenously into severe combined immunodeficient and nonobese diabetic (NOD/SCID) mice, have been used to follow up the expansion of hematopoietic populations that had been cultured ex vivo from cord blood $(4,7)$. Such assays have revealed a discrepancy between stem cell content as predicted by the in vitro surrogate assays (LTC-IC, CAFC), and in vivo repopulating ability (9). In vitro assays can measure pluripotent cells with extensive self-renewal capacity, but they do not measure the additional properties of stem cells that contribute to their efficient homing to the bone marrow. Two such features have recently been revealed: the cell's ability to detect and respond to a chemokine gradient $(8,10)$, and its upregulation of cytoadhesion molecules that allow it to be trapped by the endothelium of the marrow sinusoids (11). Human CAFC, LTC-IC and NOD/SCID repopulating cells chemotax across membranes or endothelial barriers within 3 hours, in response to a marrow stroma-derived chemokine. Signaling by this stromal cell-derived factor-1 (SDF-1) through the CXCR4 receptor $(8,10)$ may be impaired after ex
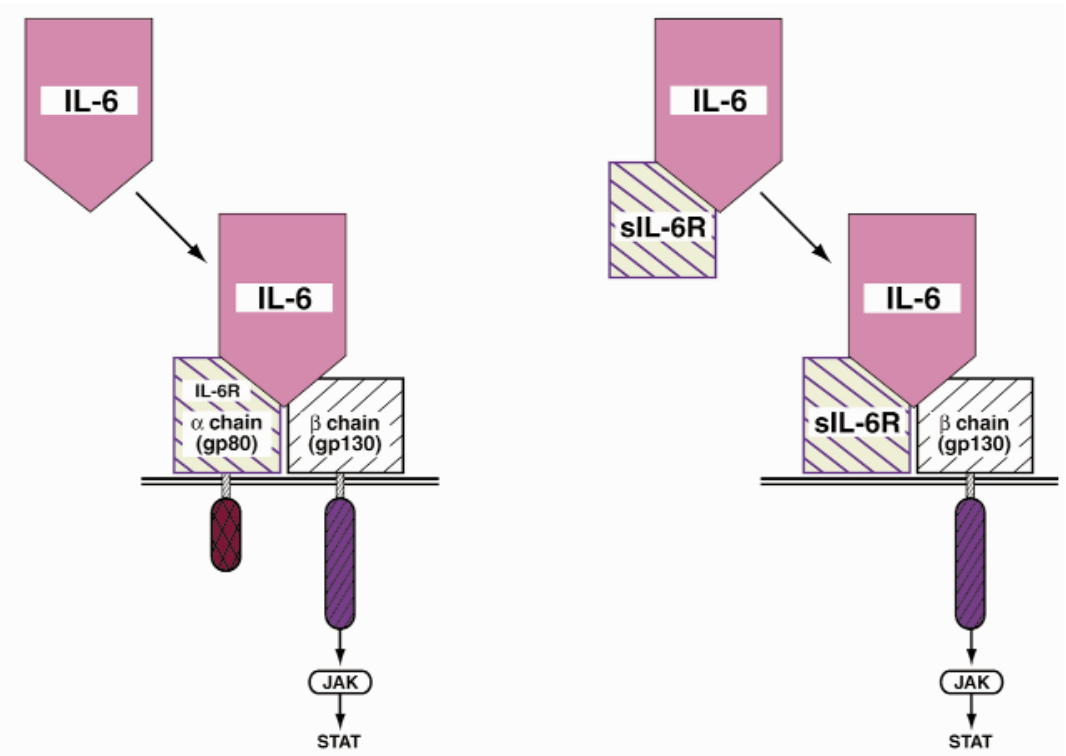

Figure 1

A schematic model of the IL- 6 receptor system. Both IL-6R $\alpha$ and gp130 belong to the type I cytokine receptor family. Many hematopoietic cells express both IL-6R $\alpha$ and gp130 as shown to the left, and the complex can bind IL- 6 and signal through gp130 with signal transduction through JAK1, JAK2, or TYK2 (shown here as JAK) and STATs -1, -3 and -5 (STAT). As shown on the right, hematopoietic stem cells may lack the IL-6R $\alpha$ and thus may be unable to bind IL- 6 to form a signaling complex through gp130. The soluble extracellular domain of the IL6R $\alpha$ can bind IL-6, forming a complex that can bind to gp130, activating signal transduction in stem cells. 
adhesion of $\mathrm{CD} 34^{+}$progenitors under shear flow into firm adhesion. Thus, impaired CXCR4 signaling or expression on cultured stem cells would be expected to reduce marrow engraftment by blunting the chemotactic response and the subsequent integrin-mediated arrest of stem cells in marrow sinusoids.

The study of Ueda et al. in this issue of JCI is the latest contribution to the 7 year saga of optimizing CB expansion (12). These authors report that a combination of SCF, FL, TPO, IL6, and a soluble form of the IL- 6 receptor (sIL-6R) was superior to other formulations for expanding CB cells with NOD/SCID engraftment potential. The proportion of human CD45 cells engrafted in the mouse marrow at 10-12 weeks was 10 fold higher with 7-day-cultured CB cells than with fresh cells, and repopulating cells were expanded 4.2-fold by limiting dilution assay. These same investigators had earlier reported potent synergism between SCF and a complex of IL- 6 and sIL-6R for cord blood progenitor expansion, and their current study extends the observed synergism to expansion of cells with in vivo stem cell potential. The rationale for the use of sIL-6R was based on the earlier observation (13) that human $\mathrm{CD} 34^{+}$LTC-ICs expressed the signaling component of the IL- 6 receptor pathway but not the ligand-binding $\alpha$-chain of the IL-6R (Figure 1). Enhanced signaling through gp130 on stem cells may be advantageous in 2 ways: favoring self-renewal and upregulating CXCR4, which favors efficient homing to the marrow. A very high concentration of sIL-6R $(1,000 \mathrm{ng} / \mathrm{mL})$ was needed to obtain the expansion reported. Fischer et al. (14) have developed an IL-6 and sIL-6R fusion protein that was fully active at a 100- to 1,000-fold lower concentration than the combined unlinked IL- 6 and IL-6R for stimulating hematopoietic progenitor expansion.

How soon can these improvements be translated into the clinic? Currently, clinical trials of ex vivo expanded cord blood are under way in the United States and Europe, using CD $34^{+}$selected cells expanded in Teflon culture bags, or nonselected CB mononuclear cells expanded in bioreactors. Various cytokine combinations are being tested, including FL, SCF, Tpo, and/or G-CSF or GM-CSF/IL-3 fusion protein. Upon availability of clinical grade IL-6/sIL$6 \mathrm{R}$, it will be a relatively simple task to address whether its addition to the cytokine cocktails in current use for clinical expansion would lead to improved stem cell engraftment.

1. Rubinstein, P., et al. 1998. Outcomes among 562 recipients of placental blood transplants from unrelated donors. N. Engl. J. Med. 339:1565-1577. 2. Broxmeyer, H.E., et al. 1992. Growth characteristics and expansion of human umbilical cord blood and estimation of its potential for transplantation in adults. Proc. Natl. Acad. Sci. USA. 89:4109-4113.

3. Moore, M.A.S., and Hoskins, I. 1994. Ex vivo expansion of cord blood-derived stem cells and progenitors. Blood Cells. 20:468-481.
4. Conneally, E., Cashman, J., Petzer, A., and Eaves, C. 1997. Expansion in vitro of transplantable human cord blood stem cells demonstrated using a quantitative assay of their lympho-myeloid repopulating activity in nonobese diabetic-scid/scid mice. Proc. Natl. Acad. Sci. USA. 94:9836-9841.

5. Ohmizono, Y., et al. 1997. Thrombopoietin augments ex vivo expansion of human cord bloodderived hematopoietic progenitors in combination with stem cell factor and flt 3 ligand. Lenkemia. 11:524-530.

6. Piacibello, W., et al. 1997. Extensive amplification and self-renewal of human primitive hematopoietic stem cells from cord blood. Blood. 89:2644-2653.

7. Piacibello, W., et al. 1999. Engraftment in nonobese diabetic severe combined immunodeficient mice of human CD34+ cord blood cells after ex vivo expansion: evidence for the amplification and self-renewal of repopulating stem cells. Blood. 93:3736-3749.

8. Jo, D.-Y., Raffi, S., Hamada, T., and Moore, M.A.S 2000. Chemotaxis of primitive hematopoietic cells in response to stromal cell-derived factor-1. J. Clin. Invest. 105:101-111.

9. Gan, O.I., Murdoch, B., Larochelle, A., and Dick, J.E. 1997. Differential maintenance of primitive human SCID-repopulating cells, clonogenic progenitors and long-term culture-initiating cells after incubation on human bone marrow stromal cells. Blood. 90:641-650.

10. Peled, A., et al. 1999. Dependence of human stem cell engraftment and repopulation of NOD/SCID mice on CXCR4. Science. 283:845-848.

11. Peled, A., et al. 1999. The chemokine SDF-1 stimulates integrin-mediated arrest of CD34+ cells on vascular endothelium under shear flow. J.Clin. Invest. 104:1199-1211.

12. Ueda, T., et al. 2000. Expansion of human NOD/SCID-repopulating cells by stem cell factor, Flk2/Flt3 ligand, thrombopoietin, IL-6 and soluble IL-6 receptor. J.Clin Invest. 105:1013-1021.

13. Tajima, S., et al. 1996. Analysis of interleukin 6 receptor and gp130 expressions and proliferative capability of human CD34+ cells. J. Exp. Med. 184:1357-1346.

14. Fischer, M., et al. 1997. A bioactive designer cytokine for human hematopoietic progenitor cell expansion. Nat. Biotechnol. 15:142-145. 